\title{
Mechanical Properties of Rice Husk Ash/Polypropylene Composites
}

\author{
M. Y. Ahmad Fuad, ${ }^{* \dagger}$ Z. Ismail, ${ }^{*}$ M. S. Mansor, ${ }^{*}$ \\ Z. A. Mohd IshaK, ${ }^{* *}$ and A. K. Mohd OMar** \\ * Plastics Technology Centre, Standards and Industrial Research Institute of Malaysia (SIRIM), \\ P. O. Box 7035, 40911, Shah Alam, Malaysia \\ ** School of Industrial Technology, Universiti Sains Malaysia, \\ 11800, Penang, Malaysia
}

(Received March 2, 1995)

\begin{abstract}
The technical viability of using rice husk ashes (RHA) as alternative fillers in polypropylene was studied. Three types of RHA, white rice husk ash (WRHA), black rice husk ash (BRHA) and amorphous rice husk ash (AMRHA) at $10-40 \mathrm{wt} \%$ filler loading were investigated. The RHA composites were compounded by twin screw compounders and the mechanical properties of the composites evaluated. Incorporation of the RHA fillers increased the flexural modulus of the composites, particularly the BRHA composities. Modulus of the RHA composites were found to be in good agreement with theoretical values predicted by equation of Lewis and Nielsen. The increase in modulus was offset by lowering of the tensile strength, elongation at break and impact properties. Theoretical treatments of the ultimate tensile strength performed based on the model proposed by Nicolai and Nicodemo showed reasonably good agreement. The mechanical properties of the RHA composites were of comparable values with the prepared polypropylene composites filled with commercial Neuburg silica. Transformation of the crystalline RHA to amorphous RHA resulted in composites with improved tensile strength. No coupling agent was used in this study.

KEY WORDS Rice Husk Ash / Filler / Polypropylene / Composites /

Mechanical Properties /
\end{abstract}

Incorporation of fillers into a polymer is known to cause substantial changes to the mechanical properties of the composities. As polymer composites are increasingly used in various mechanical applications, the mechanical properties are perhaps the most basic and important behaviors that need to be investigated in order to evaluate the performance of the composities when they are subjected to stress.

The basic characterization of the mechanical properties are usually determined by tests resulting in various deformation versus stress dependencies, for instance the stressstrain diagrams. Examinations of such dependencies yield mechanical characteristics in term of modulus of elasticity, ultimate tensile strength, impact resistance, etc. The term mechanical is applied for this category of properties because they are often used to indicate the suitability of a material for use in mechanical applications, parts that carry a loak, absorb shock, etc. ${ }^{1}$

The principal objective of this study is to investigate the technical viability of using the rice husk ashes which are usually regarded as waste products as alternative low cost fillers in polypropylene. In this investigation, the mechanical properties of the polypropylene/ rice hush ash (RHA) composites are investigated in term of their modulus, tensile and impact properties. Modulus is a measure of

* To whom correspondence should be addressed. 
the stiffness of a material subjected to shear loading. Addition of filler normally improves the stiffness of the composities. Tensile test is among the most widely employed test method to characterize the mechanical properties of composites. From such a test, enomorous amount of important information may be obtained regarding the material's elastic properties, the character and extent of its plastic deformation, and its yield and tensile strengths and toughness. Impact test, to certain extent, indicates material toughness and resistance to impact forces. The impact strengths, though of no particular design value, are important because they can be used to compare relative response of different materials. The evaluated properties of the RHA composities are subsequently compared to those of commercial Neuburg silica filler to gauge the performance of the RHA fillers.

\section{EXPERIMENTAL}

\section{Materials}

Black rice husk ash (BRHA) was obtained from a rice mill in Sekincan, state of Selangor and the white rice husk ash (WRHA) from another mill, further north in Bagan Serai, state of Perak, Malaysia. The former RHA was obtained from a burner while the latter was collected from open air burning sites outside the mills. The polypropylene used was Propelinas 600G (homopolymer) from Polypropylene (M) Sdn. Bhd. with density, melt index and isotactivity specified to be $0.9 \mathrm{~g} \mathrm{~cm}^{-3}, 12 \mathrm{~g}$ per $10 \mathrm{~min}$ and $94 \%$, respectively.

A commercial silica filler was also compounded into the polypropylene and the mechanical properties of the composites were compared. The silica was Neuburg silica, a natural occuring inorganic mineral silica (grade Sillitin V85) quarried by Hoffman Mineral in Neuburg, Germany. The comparative study also include surface-modified RHA (amorphous RHA), i.e., the crystalline white RHA which has been converted by chemical means in the laboratory to an amorphous state. ${ }^{2}$

\section{Characterizations of RHA Fillers}

The black and white RHAs were reduced to fine particles by means of a ball mill. The black RHA was ground using a William Boulton Vibro Energy Mill while the white RHA utilized a bigger pilot plant scale, customdesigned ball mill. The chemical composition was characterized using atomic absorption spectroscopy and thermogravimetry techniques. The particle size distribution was determined by a Horiba Capa-700 particle analyzer. The surface area was calculated by the Brunauer-Emmett-Teller (BET) method using Micromeritics FlowSorb II 2300. The density of the RHA particles was measured using a glass pyknometer, based on a modified technique described elsewhere. ${ }^{3}$

To determine whether the RHA is crystalline or amorphous in nature, wide angle X-ray diffraction measurements were carried out on the RHA samples using Rigaku X-ray diffraction equipment (Model RAD-IIC) with a Geigerflex scintillation counter and recorder. Radial scans of intensity $(I)$ versus diffraction angle $(2 \theta)$ were recorded in the range $5^{\circ}-80^{\circ}$ of $2 \theta$ using $\mathrm{Cu}-K_{\alpha}$ radiation. The scan speed was $10^{\circ}$ per minute and a nickel filter was used.

The white RHA filler was subjected to the FTIR analysis to characterize the silica related groups present in the filler material. A Perkin Elmer 1610 FTIR spectrophotometer was employed for this investigation. Since the WRHA is in powder form, it cannot be analyzed directly by the common transmission method and an alternative method technique was resorted to i.e., by the diffuse reflectance technique. The RHA powder was placed in a sample cup of a diffuse reflectance accessory (DRIFT of Spectra Tech) and scanned for 16 times to reduce the noise to signal ratio from 4000 to $400 \mathrm{~cm}^{-1}$.

\section{Processing}

The RHA fillers were compounded into 
polypropylene by means of a Brabender DSK 42/7 twin screw compounder having barrel temperatures of 190,200 , and $210^{\circ} \mathrm{C}$ from feeding zone to the die zone, respectively. The compounds were extruded through a twin 4-mm rod die into a water bath, pulled and plletised by an on-line pelletiser. The compounded samples were prepared into test specimens by injection moulding technique. The machine used was a 20-tonne Battenfeld BA 200 CD Plus machine, with UNILOG 4000 control system (closed-loop control). Moldings were prepared using $130 \mathrm{~kg} \mathrm{~cm}^{-2}$ injection pressure, barrel temperature profile ranging from 175 to $195^{\circ} \mathrm{C}$ and cooling time of 17 seconds. A test specimen mold from Mastermould Inc. having cavities for tensile specimen according to ASTM D638 Type 1 and a rectangular bar, $125 \mathrm{~mm} \times 12.5 \mathrm{~mm} \times 3.13 \mathrm{~mm}$, was employed for production of the test specimens.

\section{Mechanical Tests}

Flexural test was carried out according to ASTM D790-Test Method 1, Procedure A, i.e., three-point loading system utilising centre loading. The support span was $50 \mathrm{~mm}$ whilst the diameter of the loading nose and supports were $20 \mathrm{~mm}$ and $10 \mathrm{~mm}$, respectively. Test was conducted at a test speed of $2 \mathrm{~mm} \mathrm{~min}^{-1}$ on a Lloyd L1000S tensile/compression machine. Since the modulus was determined between small initial deflections, to ensure good accuracy, a low force load cell $(100 \mathrm{~N})$ was used. Tensile tests were carried out on the same machine, using a load cell of $5 \mathrm{kN}$ and an Infrared non-contacting extensometer for elongation determination. The gauge length was $50 \mathrm{~mm}$ (ASTM D 638-Type I) and the speed of testing $5 \mathrm{mmmin} .^{-1}$ Tests were performed as specified in ASTM D 638. Izod test specimens were prepared from the injected rectangular bar. Notching $\left(45^{\circ}\right)$ was carried out on the specimens using Davanport notch cutting apparatus. Izod impact test was performed using a 2-Joule hammer (Ceast Izod tester). The test was conducted based on ASTM D 256-88.

\section{RESULTS AND DISCUSSION}

\section{RHA Fillers Characterizations}

The chemical and physical properties of the RHA fillers as characterized by the techniques descibed in are presented in Table I. It may be observed that the white RHA consists predominantly of silica $(96 \%)$ while the black RHA contain only about $54 \%$ silica but a substantially high carbon content ( $44 \%$ ) as indicated by the loss on ignition value. Apart from silica and carbon, the other metal oxides such as $\mathrm{CaO}, \mathrm{MgO}, \mathrm{Fe}_{2} \mathrm{O}_{3}, \mathrm{~K}_{2} \mathrm{O}, \mathrm{Na}_{2} \mathrm{O}, \mathrm{Al}_{2} \mathrm{O}_{3}$, and $\mathrm{P}_{2} \mathrm{O}_{5}$ that were present at comparable levels make up the rest of the constituents. The white RHA has slightly larger median particle size $(6.6 \mu \mathrm{m})$, smaller surface area $\left(1.4 \mathrm{~m}^{2} \mathrm{~g}^{-2}\right)$ and is more dense than the black RHA.

Figure 1 shows the spectra of white RHA filler, neat polypropylene and a typical WRHA composite. The quality of the WRHA spectrum obtained by the diffuse reflectance technique and subjected to the Kubelka-Munk transformation (to get the equivalent of transmission spectrum) is not as good as the thin films' transmission spectra of the neat polypropylene

Table I. Chemical and physical properties of white and black rice husk ashes

\begin{tabular}{lcc}
\hline \multicolumn{1}{c}{ Property } & White RHA & Black RHA \\
\hline Chemical composition $/ \%$ & & \\
$\mathrm{CaO}$ & 0.36 & 0.12 \\
$\mathrm{MgO}$ & 0.16 & 0.078 \\
$\mathrm{Fe}_{2} \mathrm{O}_{3}$ & 0.041 & 0.022 \\
$\mathrm{~K}_{2} \mathrm{O}$ & 0.69 & 0.95 \\
$\mathrm{Na}_{2} \mathrm{O}$ & 0.034 & 0.018 \\
$\mathrm{Al}_{2} \mathrm{O}_{3}$ & 0.025 & 0.023 \\
$\mathrm{P}_{2} \mathrm{O}_{5}$ & 0.57 & 0.27 \\
$\mathrm{SiO}_{2}$ (silica) & 96.20 & 53.88 \\
$\mathrm{Loss}_{0}$ on ignition (LOI) & 1.62 & 44.48 \\
$\mathrm{Physical}$ properties & & \\
$\mathrm{Particle} \mathrm{size} / \mu \mathrm{m}_{\text {Surface area } / \mathrm{m}^{2} \mathrm{~g}}^{-1}$ & 6.6 & 19.5 \\
Density $/ \mathrm{g} \mathrm{cm}$ & 1.4 & 26.8 \\
& 2.2 & 1.8 \\
\hline
\end{tabular}




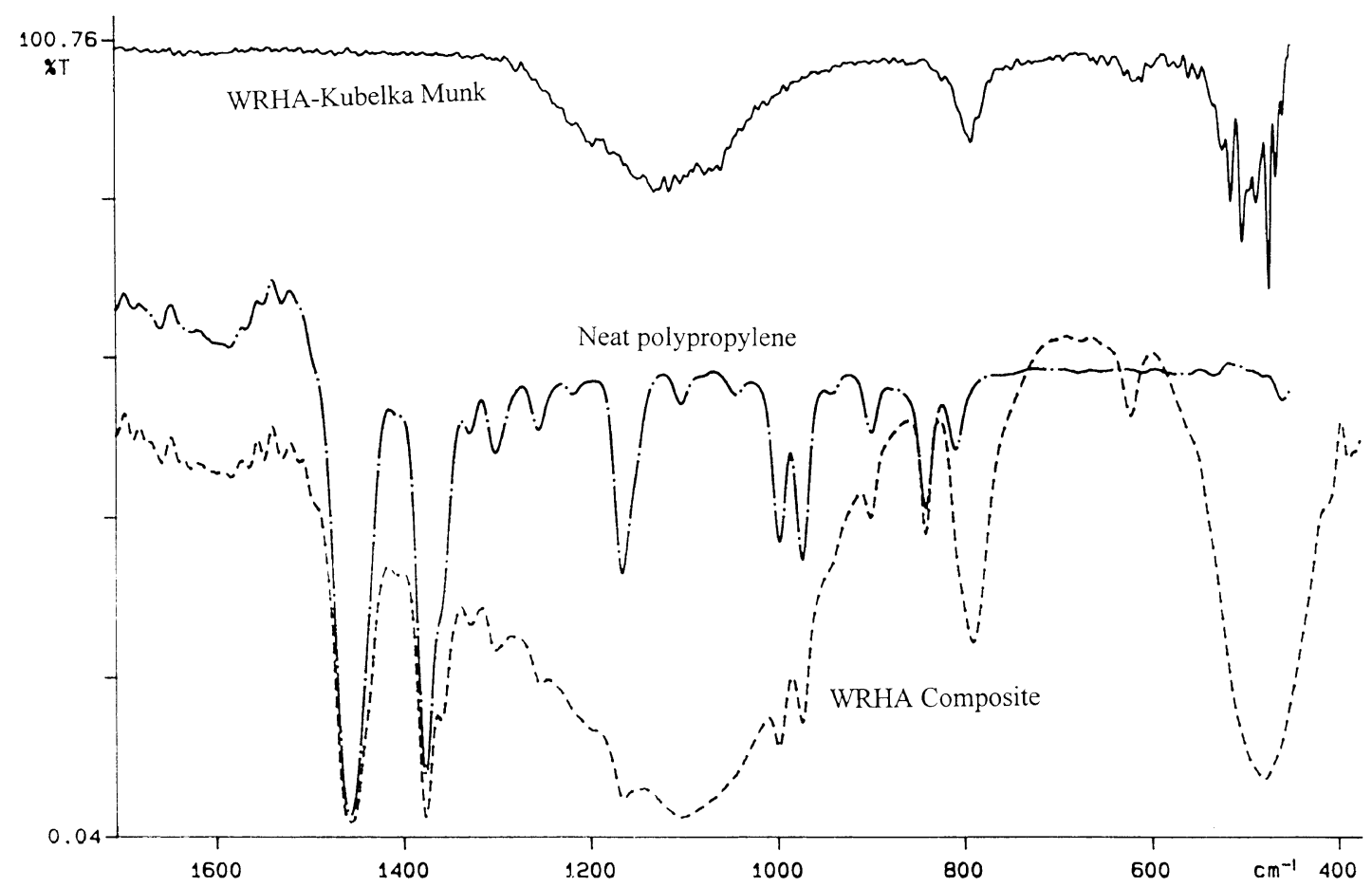

Figure 1. The FTIR spectra of WRHA (top), unfilled polypropylene (middle), and WRHA composite (bottom) at $20 \%$ loading.

(middle spectrum) and the WRHA composite (lowermost spectrum). Anyway the peaks are still observable and may be used as references when studying the spectra of the WRHA composites later on. Being a relatively heavy atom, the infrared silica bands appear more towards the far infrared region (lower wave number). Four major peaks may be associated to the WRHA, viz. peaks at $480,621,790$, and $1130 \mathrm{~cm}^{-1}$. These peaks may be assigned probably to the following respective bonds: $\mathrm{Si}-\mathrm{O}_{2}, \mathrm{SiH}_{3}, \mathrm{Si}-\left(\mathrm{CH}_{3}\right)_{x}$, and $\mathrm{Si}-\mathrm{O}-\mathrm{Si}^{4}{ }^{4}$ Similar peaks are not present in the spectrum of the neat polypropylene. The peaks are, however, more prominent and sharper in the WRHA composite $(20 \%$ filler content) spectrum as shown by the lowermost spectrum.

FTIR analysis was not conducted on the BRHA filler since the carbon component of the filler induces a total abosorption of the incident infrared energy. Moreover, in using the diffuse reflectance technique, much of the initial radiation has been lost in passing through the various stages of the accessory and that only about $15 \%$ of the energy was available to start with. The high carbon content in the RHA virtually cut off all the available energy.

Figures $2 \mathrm{a}$ and $\mathrm{b}$ show the X-ray diffractograms of the white and black RHA fillers, respectively. Sharp peaks in the former indicates the crystalline nature of the white RHA while absence of any distinct peak shows that the black RHA is amorphous in nature. X-Ray diffractogram for Neuburg silica and AMRHA fillers are shown in Figures $2 c$ and d.

\section{Mechanical Tests Results}

The overall results for the mechanical properties of the RHA composites are summarized in Table II. The detail analyses of tensile, flexural and Izod impact tests results may be 
M. Y. A. FUAD et al.
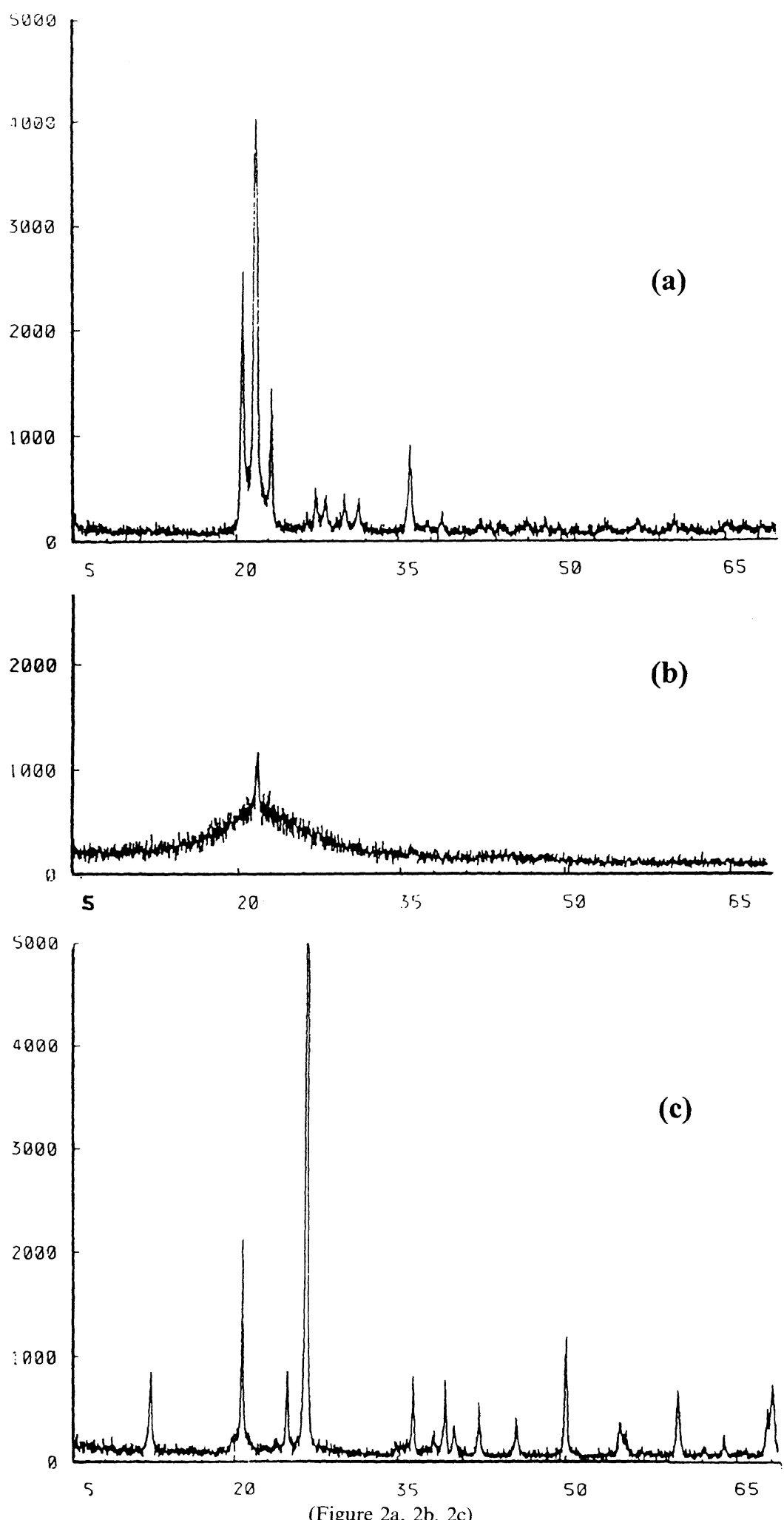


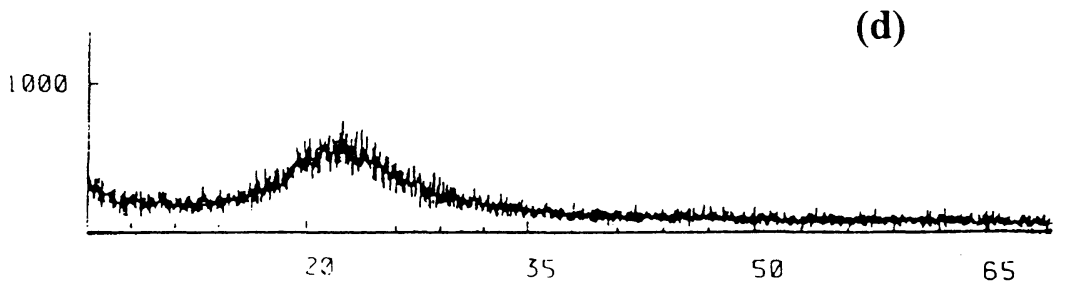

(Figure 2d)

Figure 2. The X-Ray diffractograms of (a) WRHA, (b) BRHA fillers, (c) Neuburg silica, and (d) AMRHA particles. Sharp peak indicate crystalline nature of WRHA and absence of such peak shows that BRHA is amorphous.

Table II. Results of mechanical tests on the RHA composites

\begin{tabular}{|c|c|c|c|}
\hline Property & $\begin{array}{c}\text { Filler } \\
\text { content } / \%\end{array}$ & WRHA & BRHA \\
\hline \multirow{5}{*}{$\begin{array}{l}\text { Flexural } \\
\text { modulus/MPa }\end{array}$} & 0 & \multicolumn{2}{|c|}{1579} \\
\hline & 10 & 1639 & 1897 \\
\hline & 20 & 1936 & 2306 \\
\hline & 30 & 2292 & 2639 \\
\hline & 40 & 2544 & 3124 \\
\hline \multirow{5}{*}{$\begin{array}{c}\text { Tensile strength } \\
\text { at yield/MPa }\end{array}$} & 0 & \multicolumn{2}{|c|}{34.5} \\
\hline & 10 & 31.2 & 28.9 \\
\hline & 20 & 27.0 & 26.9 \\
\hline & 30 & 24.9 & 23.9 \\
\hline & 40 & 21.8 & 23.5 \\
\hline \multirow{5}{*}{$\begin{array}{l}\text { Tensile strength } \\
\text { at break/MPa }\end{array}$} & 0 & \multicolumn{2}{|c|}{26.3} \\
\hline & 10 & 25.8 & 24.8 \\
\hline & 20 & 22.5 & 23.8 \\
\hline & 30 & 21.1 & 22.6 \\
\hline & 40 & 19.1 & 22.1 \\
\hline \multirow{5}{*}{$\begin{array}{l}\text { Elongation } \\
\text { at break } / \%\end{array}$} & 0 & \multicolumn{2}{|c|}{$>600 \%$} \\
\hline & 10 & $>600 \%$ & 26 \\
\hline & 20 & 127 & 11.2 \\
\hline & 30 & 50.6 & 6.4 \\
\hline & 40 & 22.1 & 1.7 \\
\hline \multirow{5}{*}{$\begin{array}{l}\text { Izod impact } \\
\text { strength } / \mathrm{Jm}^{-1}\end{array}$} & 0 & \multicolumn{2}{|c|}{110} \\
\hline & 10 & 72.6 & 45.8 \\
\hline & 20 & 68.4 & 42.3 \\
\hline & 30 & 54.4 & 28.5 \\
\hline & 40 & 50.0 & 24.8 \\
\hline
\end{tabular}

elucidated as follows.

\section{Flexural Modulus}

The flexural modulus of the RHA composites are shown in Figure 3. As expected the

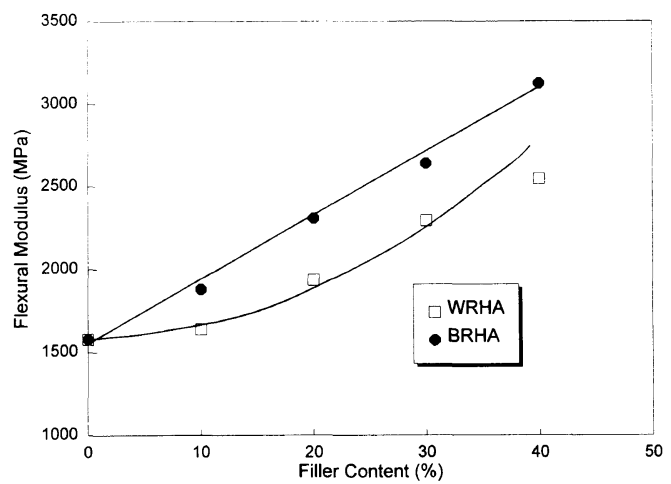

Figure 3. Effect of filler loading on the flexural modulus of the RHA composites.

modulus, which indicates material stiffness, increases steadily with greater filler content. The BRHA composite shows relatively higher stiffness than WRHA composite.

Modulus of composities has been represented by a number of equations, the first and simplest being from Einsten which may be rewritten as

$$
E_{R}=1+2.5 \phi_{\mathrm{f}}
$$

where $E_{\mathrm{R}}$ is relative modulus (of composite to polymer) and $\phi_{\mathrm{f}}$ is volume fraction of filler. The equation was further developed by Guth ${ }^{5}$ to be as follow;

$$
E_{\mathrm{R}}=1+2.5 \phi_{\mathrm{f}}+14.1 \phi_{\mathrm{f}}^{2}
$$

Kerner $^{6}$ then deduced an elaborate and versatile equation to describe the reinforcing action of the spherical filler particles as shown below: 


$$
E_{\mathrm{R}}=\frac{\frac{\phi_{\mathrm{f}} G_{\mathrm{f}}}{\left(7-5 v_{p}\right) G_{\mathrm{p}}+\left(8-10 v_{\mathrm{p}}\right) G_{\mathrm{f}}}+\frac{\phi_{\mathrm{p}}}{15\left(1-v_{\mathrm{p}}\right)}}{\frac{\phi_{\mathrm{p}} G_{\mathrm{f}}}{\left(7-5 v_{\mathrm{p}}\right) G_{\mathrm{p}}+\left(8-10 v_{\mathrm{p}}\right) G_{\mathrm{f}}}+\frac{\phi_{\mathrm{p}}}{15\left(1-v_{\mathrm{p}}\right)}}
$$

where $\phi_{\mathrm{f}}$ and $\phi_{\mathrm{p}}$ are volume fractions of filler and polymer respectively; $G_{\mathrm{f}}$ and $G_{\mathrm{p}}$ are shear moduli of filler and polymer respectively, and $v_{\mathrm{p}}$ is the poisson ratio of the polymer.

Since many fillers are much more rigid than matrices, eq 3 may be simplified to:

$$
E_{\mathrm{R}}=1+\phi_{\mathrm{f}} / \phi_{\mathrm{p}}\left\{\left[15\left(1-v_{\mathrm{p}}\right)\right] /\left(8-10 v_{\mathrm{p}}\right)\right\}
$$

Lewis and Nielsen further modified the Kerner equation by taking into account the maximum packing fraction, $\phi_{\mathrm{m}}$ of the filler particles, interparticle interaction and the relative modulus of the two constituents. ${ }^{7}$

$$
E_{\mathrm{R}}=\left(1+A B \phi_{\mathrm{f}}\right) /\left(1-B \Psi \phi_{\mathrm{f}}\right)
$$

where

$$
\begin{aligned}
A & =\left(7-5 v_{\mathrm{p}}\right) /\left(8-10 v_{\mathrm{p}}\right) \\
B & =\left[\left(G_{\mathrm{f}} / G_{\mathrm{p}}\right)-1\right] /\left[\left(G_{\mathrm{f}} / G_{\mathrm{p}}\right)+A\right] \\
\Psi & =1-\left[\left(1-\phi_{\mathrm{m}}\right) / \phi_{\mathrm{m}}^{2}\right] \phi_{\mathrm{f}}
\end{aligned}
$$

$A$ is a constant related to Einstein coefficient and is a function of filler's geometry, $B$ is related to relative moduli of filler and polymer and $\Psi$ is a reduced concentration term dependent on the maximum packing fraction $\phi_{\mathrm{m}}$ of the filler in the polymer. $G_{\mathrm{f}}$ and $G_{\mathrm{p}}$ are moduli of filler and polymer matrix, respectively.

For low aspect ratio fillers like the white and black RHAs, the Lewis and Nielsen equation seems to be more applicable and will be used for calculating the theoretical values of the modulus. A few assumptions have to be made to qualify the RHA composites for such calculation. Firstly it will be assumed that the RHA particles are spherical in shape, well dispersed and experience random packing. As such the value of the maximum packing fraction $\phi_{\mathrm{m}}$ will be 0.64 as used by Lewis and Nielsen while deducing the above series of equations. Secondly, the modulus of the white RHA is assumed to be equivalent to the literature value for the modulus of silica, i.e., $G_{\mathrm{f}}=72 \mathrm{GP}^{8}{ }^{8}$ Using the above values and substituting in equations ( 5 and $5 \mathrm{a}-\mathrm{c}$ ), the theoretical moduli for various filler loading may be calculated. Figure 4a compares the theoretical Nielsen's curve using the values of $A$ as 1.5 and 1.0 with the experimental values of the white RHA composites. It may be observed that the most of the experimental values lie between the two theoretical curves indicating good agreement especially if $A=1.0$.

For the black RHA filler, another assumption has to be made. Since the basic composition of the filler is made up of a mixture of approximately $54 \%$ silica and $44 \%$ of carbonaceous material, the modulus is expected to be considerably lower. For the sake of calculation, the modulus of the black RHA filler is assumed to be half of the modulus of the white RHA, i.e., $G_{\mathrm{f}}=36 \mathrm{GPa}$. At the same time, the filler is also assumed to be less spherical due to its impure nature and thus assigned a higher value of the constant $A$. Similar moduli calculations were performed using the values of 2.0 and 2.5 for $A$ and the curves are shown in Figure $4 \mathrm{~b}$. The curve with $A=2.5$ seems to give better fit to the experimental results. Overall, we may say that the experimental values for the moduli of both the white and black RHA composites agree reasonably well with the predicted theoretical values as proposed by Lewis and Nielsen.

Jilken obtained good agreement with Lewis and Nelsen equation for their experimental results on dolomite (filler modulus approx. $35 \mathrm{GPa}$ ) by letting $A=3 .^{9}$ Likewise reasonably close agreement was obtained when they relates their experimental data on mica filler (flakes) with Padawar-Beecher equation. Bigg used 3.0 as the value for $A$ and assumed $\phi_{\mathrm{m}}$ is equal to 0.5 to draw up the theoretical Nielsen's curve that compares well with his thermoplastic composites filled with talc, silicon carbide and aluminium flake fillers. ${ }^{10}$ 

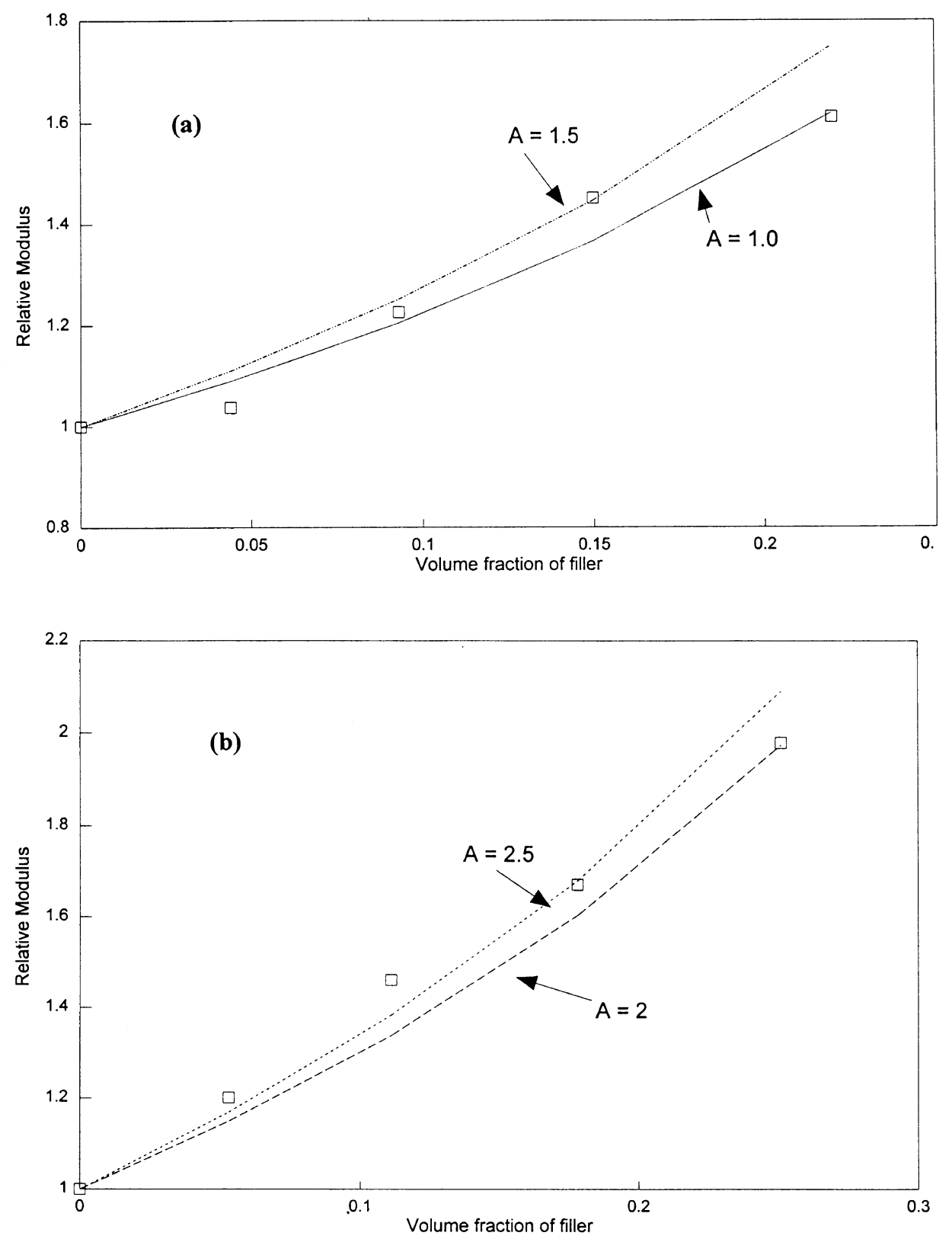

Figure 4. Comparison between the experimental data and theoretical modulus values for (a) WRHA and (b) BRHA composites. Experimental data are represented by open square symbols while the theoretical values are represented by the continuous lines. $A$ is a constant related to filler's geometry.

\section{Tensile Properties}

Fibre type fillers normally improve tensile strength of the composite as the fibres are able to support stresses transferred from the poly- mer via the interfacial region. For irregularly shaped particulate fillers, strength of the composites frequently decreases due to the inability of the fillers to support stresses transfer 
from the polymer matrix. In this study, the ultimate (yield) tensile strength of both BRHA and WRHA composites decreases steadily with increasing filler loading (Figure 5). The fact that no coupling agent was present in the composites, result in a poor adhesion of the RHA particulates to the polypropylene matrix. Weak interfacial regions imply that the transfer of stress from the polymer matrix to the filler with not be efficient. Hence the drop in the tensile strength is quite expected.

Nielsen predicted the stress-strain behavior of filled polymer as a function of filler concentration for both cases of perfect adhesion and no adhesion between filler and polymer. ${ }^{11}$ For the case of no adhesion, the filler particles cannot carry any of the load applied to the composites and the entire stress has to be carried solely by the matrix material. If the filler particles are not wetted by the matrix polymer, inevitably the filler particles and agglomerates are filled with air, introducing porosity to the internal structure of the composites that have adverse effect on the tensile strength. In addition to the effect of porosity, stress concentrations around the particles will reduce the strength even further. Thus Nielsen predicted the tensile strength to be:

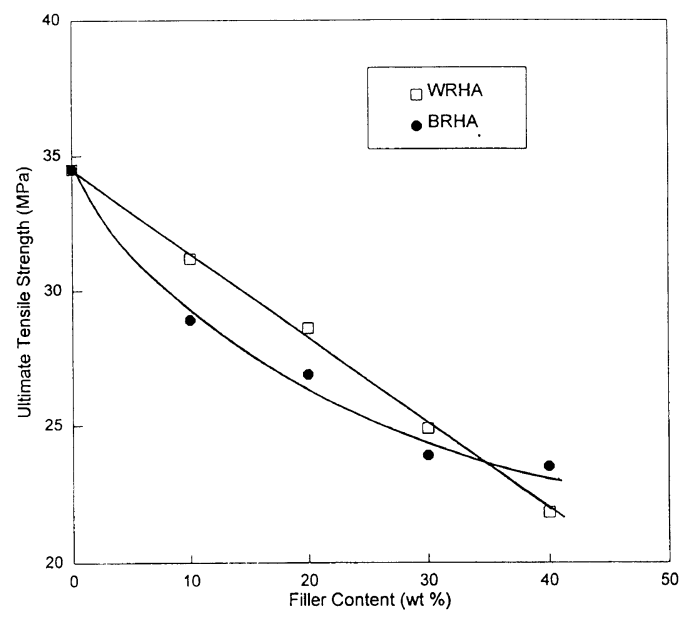

Figure 5. Effect of filler loading on the tensile strength of the RHA composites.

$$
\sigma_{\mathrm{B}}(\text { filled }) / \sigma_{\mathrm{B}}(\text { unfilled })=\left(1-\phi_{\mathrm{f}}^{2 / 3}\right) S
$$

where $S$ is the stress concentration function, $\phi_{\mathrm{f}}$ is the volume fraction of the filler and $B$ stands for "break", i.e., tensile strength (or to be precise, tensile stress) at break.

It shall be noted that the RHA composites and the neat polypropylene experience lower tensile stress at break than the yield tensile stress. Thus the yield tensile stress values are subsequently taken as the ultimate tensile strength of the materials. The above Nielsen's theory consider the case of tensile stress at break, and not the yield tensile strength. The theoretical values for the tensile stress at break for both white and black RHA composites were calculated and plotted using eq 6 for the case of $S=1$, i.e., assuming no stress concentration factor. The theoretical curves for both composites (Figures 6a and b) show that the ten-
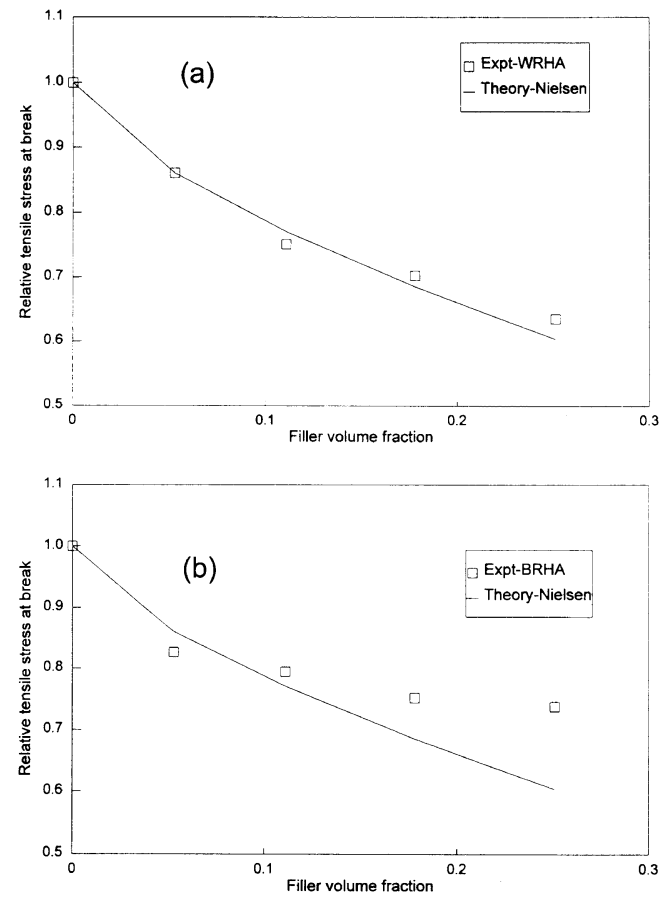

Figure 6. Comparison between the experimental data and theoretical tensile stress at break (after Nielsen, 1966) for (a) WRHA and (b) BRHA composites. Experimental data are represented by open square symbols while the theoretical values are represented by the continuous. 
sile strength decreases rapidly with increasing filler content. When the experimental tensile strength values of the composites were fitted to the theoretical curves, they showed reasonably good agreement, particularly for the case of white RHA composite (Figure 5). Of course, the assumptions used in the previous modulus study (spherical particles, well dispersed and random packed) are also applicable in this case too.

Using an alternate model based on the work of Smith, Nicolais and Narkis derived independently a very similar equation to describe the dependence of yield stress of particulatefilled composites on the volume fraction of filler. $^{12}$

$$
\sigma_{\mathrm{yc}} / \sigma_{\mathrm{yp}}=\left(1-1.21 \phi_{\mathrm{f}}^{2 / 3}\right)
$$

where $\sigma_{\mathrm{yc}}$ and $\sigma_{\mathrm{yp}}$ refer to the yield stresses of the composite and unfilled polymer respectively. Nicolais and Nicodemo made use of eq 7 to estimate the lower bound of tensile strength of a filled polymer. ${ }^{13}$ The tensile strength of the composite, $\sigma_{\mathrm{c}}$ may be related to the matrix tensile strenfth, $\sigma_{\mathrm{p}}$ simply by:

$$
\sigma_{\mathrm{c}}=\sigma_{\mathrm{p}}\left(1-1.21 \phi_{\mathrm{f}}^{2 / 3}\right)
$$

where the constants 1.21 and $2 / 3$ are related to stress concentration and geometry of the filler, respectively. For spherical fillers with no adhesion to the polymer matrix, the value of first constant has been found equal to 1.21. For the case of som adhesion, it becomes smaller than 1.21. The second constant is equal to 1 if the material fails by planar fracture and $2 / 3$ if failure is by random fracture.

While both modes of fractures have been observed, random fracture is more common. ${ }^{10}$ For the purpose of calculation, if the RHA particles are assmumed to be spherical and the composites failed by random fractures, the lower bound curves (LBC) for each RHA composite will be as shown in Figures $7 \mathrm{a}$ and b. The discrepancy observed between the theoretical lower bound curves and the experimental curves is probably due to the assump- tion that the RHA particles are spherical and exist as discrete particles while in reality they have irregular shapes and are in the form of agglomerates.

The upper bound of a tensile strength of a polymer filled with spherical particles has been derived by Leidner and Woodhams as:

$$
\sigma_{\mathrm{c}}=\left(\sigma_{\mathrm{I}}+0.83 \tau_{\mathrm{m}}\right) \phi+\sigma_{\mathrm{p}}(1-\phi)
$$

where $\sigma_{1}$ represents the interfacial tensile strength between the polymer and filler, and $\tau_{\mathrm{m}}$ the shear strength of the polymer. ${ }^{14}$ However, the upper bound response is not examined in this study since the response assumes strong adhesion between polymer and filler. Such assumption is not valid in the present RHA composites since no coupling agent has been incorporated into the RHA composities to improve the adhesion between the RHA fillers and polypropylene matrix.
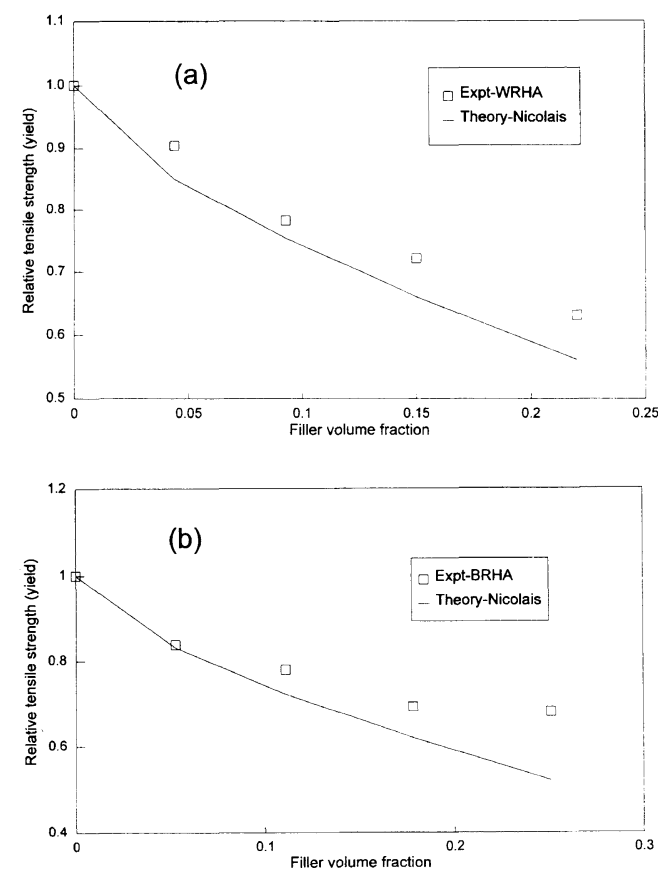

Figure 7. Comparison between the experimental data and theoretical ultimate tensile strength (after Nicolais and Nicodemo, 1973) for (a) WRHA and (b) BRHA composities. Experimental data are represented by open square symbols while the theoretical values are represented by the continuous line. 
Figure 8 shows the effect of filler loadings on the elongation at break, $\varepsilon_{\mathrm{B}}$ for the various RHA composites. The $\varepsilon_{\mathrm{B}}$ for the unfilled polypropylene and WRHA composite at $10 \%$ filler loading samples are not shown in the figure because these samples did not break till the maximum limit of the testing machine. They have $\varepsilon_{\mathrm{B}}$ values of more than $600 \%$. For the rest of the RHA composites, the expected trend is observed, i.e., the $\varepsilon_{\mathrm{B}}$ decreases rapidly with increasing filler content. The drop in the elongation at break is much more apparent in BRHA composites; where even at $10 \%$ filler loading, the $\varepsilon_{\mathrm{B}}$ was reduced from excess of $600 \%$ to $26 \%$ only. At maximum loading of $40 \%$, the BRHA composite has an extremely low $\varepsilon_{\mathrm{B}}$ of $1.7 \%$.

When subjected to stress at a low strain rate, the macromolecular chains of the polymer align themselves along the axial direction in line to the applied tensile force. Incorporation of fillers that have poor adhesion to the polymer matrix seems to cause interruption in the alignment process of the chains. When filler concentration is increased, more weak interfacial regions between the filler surface and polypropylene matrix are formed. As crack travels easier through the weaker interfacial regions, the composite therefore fractures at lower degree of elongation with increasing filler content.

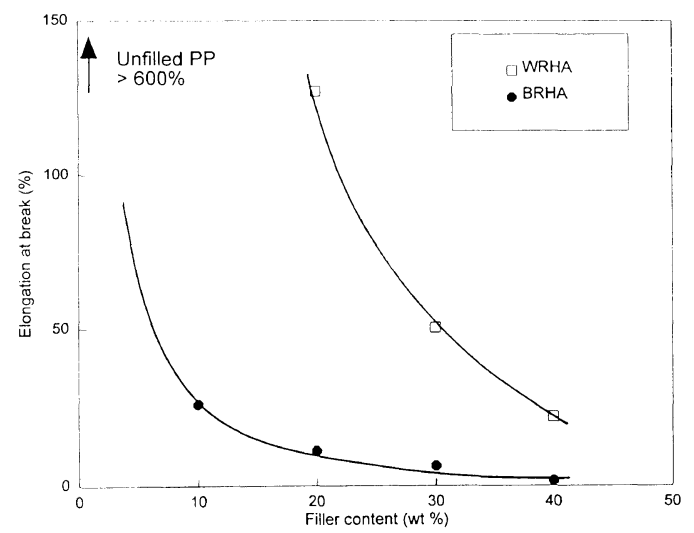

Figure 8. Effect of filler loading on the elongation at break for the RHA composites.
According to Nielsen and assuming perfect adhesion, the elongation (strain) of the polymer relative to the overall elongation of the filled system may be calculated from:

$$
\varepsilon_{\mathrm{B}}(\text { filled }) / \varepsilon_{\mathrm{B}}(\text { unfilled })=1-\phi_{\mathrm{f}}^{1 / 3}
$$

where $\varepsilon_{\mathrm{B}}$ is the elongation at break as measured in the tensile stress-strain test of the filled system. ${ }^{11}$ For the elongation at break for the case of no adhesion, he suggested combining eq 10 with another equation derived by Sato and Furukawa. Overall as depicted by Nielsen, the elongation of systems with perfect adhesion is considerably less than the corresponding systems without adhesion. If this criterion is to be applied to the elongation of the RHA composites, the BRHA composites which experience a drastic decrease in elongation with filler loading seems to have better filler to matrix adhesion than the WRHA composites.

Attempt to apply theoretical treatment to the elongation at break values met with some difficulty due to the seemingly infinite strain of the unfilled polypropylene at the test speed of choice, i.e., $5 \mathrm{~mm} \mathrm{~min}^{-1}$. At this strain rate, the unfilled polypropylene and the white RHA composite at $10 \%$ filler content yield continuously till the maximum daylight limit of the machine (percentage elongation $>600 \%$ ). Thus the value of $\varepsilon_{\mathrm{B}}$ (unfilled) could not be computed to be used for subsequent calculation of $\varepsilon_{\mathrm{B}}$ (filled). Of course the test speed of $5 \mathrm{~mm}$ $\min ^{-1}$ is far too show for the unfilled polypropylene since at this speed the material would yield unendlessly but this test speed is just right for filled materials; hence the dilemma on the choice of test speed for the comparative study. Increasing strain rate results in abrupt failure of the homopolymer, for instance at a test speed of $50 \mathrm{~mm} \mathrm{~min}^{-1}$, the percentage elongation at break for the unfilled polypropylene is about $30 \%$ only. But since the tensile-strain tests of all the RHA composites were performed at $5 \mathrm{~mm} \mathrm{~min}^{-1}, \varepsilon_{\mathbf{B}}$ (unfilled) value derived at other test speed will not be valid for subsequent stress-strain properties 
comparison purpose.

Leidner and Woodhams proposed a relationship and made several assumption to account for the ultimate strain of glass beads-filled polyester composite. ${ }^{14}$ The ultimate strain of the composite, $\varepsilon_{\mathrm{c}}$ was approximated by the derived expression:

$$
\varepsilon_{\mathrm{c}}=\left(\varepsilon_{\mathrm{m}} / \varepsilon_{\mathrm{me}}\right) \varepsilon_{\mathrm{ce}}
$$

where $\varepsilon_{\mathrm{m}}$ is the strain of the matrix at a stress equal to that carried by the matrix at the fracture point of the composite; $\varepsilon_{\mathrm{ce}}$ represents the ultimate strain of the elastic material having the ultimate strain equal to $\sigma_{\mathrm{c}}$ and $\varepsilon_{\mathrm{me}}$ the ultimate strain of the elastic material having an ultimate strength equal to $\sigma_{m}$. One of the assumptions was good matrix-filler adhesion or if the composites have poor matrix-filler adhesion, the ultimate strain was limited to low strain value only (up to about $5 \%$ only). Thus the equation is not applicable to the RHA/polypropylene system that have poor matrix-filler adhesion and excessively high strain value. Based upon these limitations, no theoretical treatment of the elongation will be presented in this study.

\section{Izod Impact Property}

Figure 9 shows the effect of filler loading on the notched Izod impact strength of filled polypropylene. Incorporation of the first $20 \mathrm{wt} \%$ of the RHA fillers into the polypropylene causes a substantial drop in the impact strength. Subsequent addition of fillers results in a more gradual decrease in impact strength. The fall in the impact strength was observed to be more prominent in the BRHA composites.

Impact failures are the result of rapid crack propagation through the material. The crack's growth rate is inversely proportional to the impact resistance of the material. For a polymer to be considered as having good impact resistance, it should be able to absorb most of the impact energy and slows the rate of crack propagation. ${ }^{10}$ According to Manson and
Sperling, filler particles tend to act as stress concentrators that supply a composite material with numerous potential sites for crack growth paricularly in the case of poor matrix to filler adhesion. ${ }^{15}$ The presence of a particulate filler normally reduced the energy required to induce and propagate a catastrophic crack.

It should be noted that the impact strength values obtained from the Izod impact test are rather sensitive to sample preparation method, sample dimensions and also to random variations from specimen to another due to the presence of microcracks and other voids. The impact strength acquired through this test is really a combination of the energy required to inititate fracture, the energy for crack propagation, the kinetic energy of the broken fragments and possible energy losses to the testing machine. Notching the samples reduce variability due to random flaws in the samples.

In the absence of any coupling agent to modify the RHA surface characteristics, poor wetting of the particles by the polypropylene matrix is expected. This gives rise to poor interfacial adhesion between the filler and the polymer matrix resulting in weak interfacial regions. During the impact test, crack travels through both the polymer as well as along the weaker interfacial regions. The latter cannot resist crack propagation as effectively as the homogenous polymer region therefore resulting in the lowering of the impact strength. Increasing the filler content merely increases

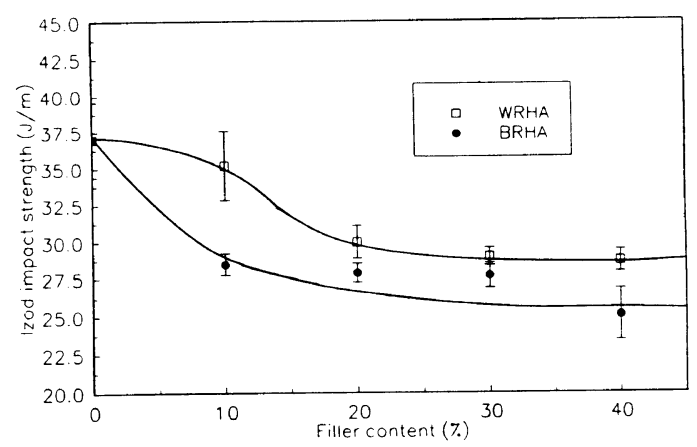

Figure 9. Effect of filler loading on the Izod impact strength of the RHA composites. 
the interfacial regions which exaggerates the weakening of the resulting composites to crack propagation. Similar trend of decreasing impact strength with increasing filler loading was also by Leidner and Woodham ${ }^{14}$ for glass beads-filled polyester, by Bigg $^{10}$ for various inorganic particulate fillers/thermoplastic composites systems and by Riley et al ${ }^{16}$ for talcfilled polypropylene composites. For calcium carbonate-filled polypropylene, however, Riley observed a contradicting effect; the calcium carbonate particles enhanced the impact strength to a value considerably greater than that of the unfilled polypropylene.

Figure 9 also indicates that the white RHA composite possess higher impact strength than the black RHA composite. This may be attributed to the greater agglomeration of the black RHA particles. Ess and Hornsby suggested that there are several adhesion forces holding the particles together in agglomerates viz. interlocking, eletrostatic, van der Waals, liquid bridge, and solid bridge forces. ${ }^{17}$ These combined interparticle forces are, however, weaker than chemical bonding of the polypropylene matrix and are therefore easier to be overcame by external mechanical energy (impact energy in this case) during fracture. Consequently BRHA particles which are believed to have a higher degree of agglomeration yield a more brittle composite and is more susceptible to cracking.

Since at present, there are no viable theoretical relationships to relate the impact strength of particulate fillers/polymer composites to the characteristics and concentration of fillers, no theroretical treatment of the experimental data would be carried out in study.

\section{Comparison to Neuburg Silica}

The performance of the RHA fillers in polypropylene is compared to a similar silica-based filler available commercially, i.e., Neuburg silica. The Neuburg silica is a natural mixture of corpuscular quartz and laminar quartz that together form a loose structure which offers particular advantages in terms of application possibilites when used as a filling material. The Neuburg silica used in this study is Sillitin B85 which has the specified particle size nearest to the white RHA particles, i.e., $2.3 \mu \mathrm{m}$. The BET surface area of the silicas was analysed to be $9.3 \mathrm{~m}^{2} \mathrm{~g}^{-1}$. Like the WRHA, the Neuburg
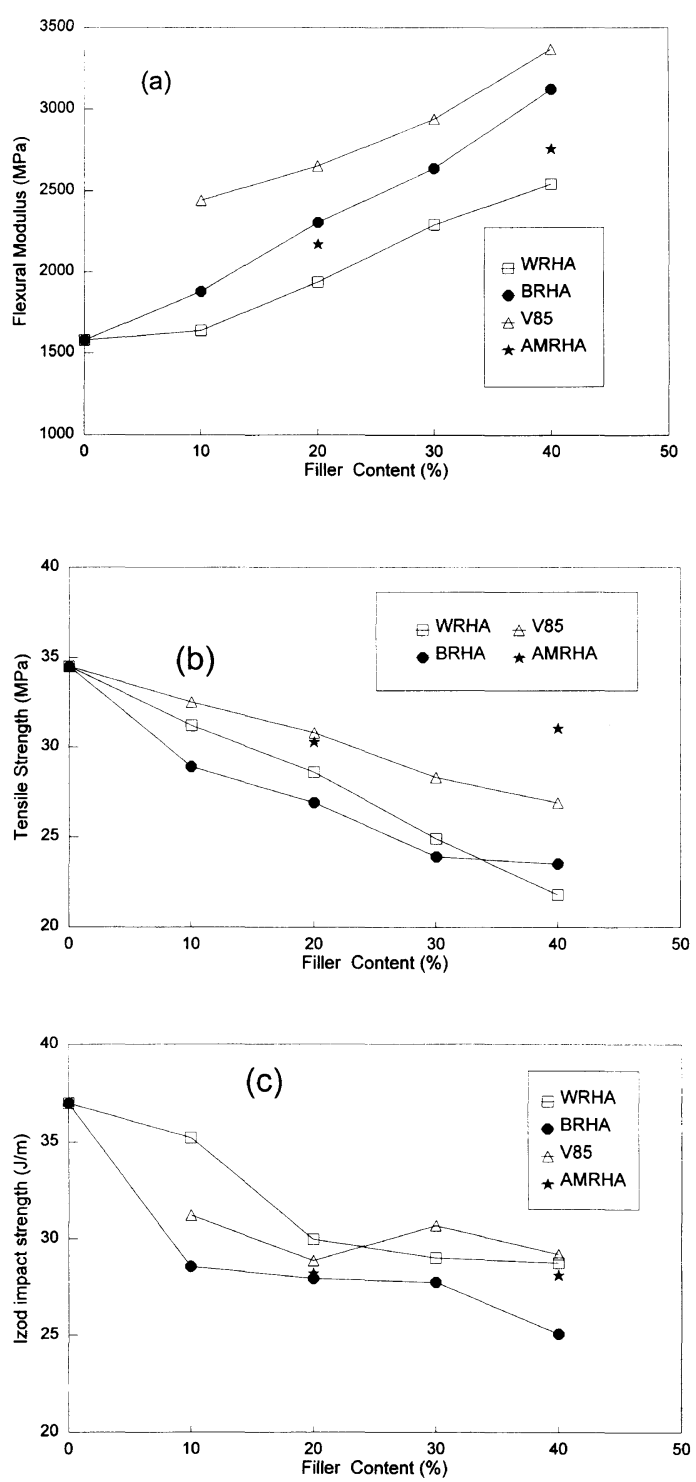

Figure 10. Comparison of the (a) flexural modulus, (b) tensile strength, and (c) Izod impact strength of the RHA composites with polypropylene composites filled with commercial Neuburg silica filler. 
silica has been shown by the X-ray scan to be crystalline (Figure 2c).

Figures $10 \mathrm{a}-\mathrm{c}$ show the mechanical properties of Neuburg silica-filled polypropylene. It may be observed that generally their properties are superior than those of the RHA composities, particularly in terms of flexural modulus and tensile strength. The impact properties are comparable to the white RHA composite and are marginally better than the black RHA composites.

\section{Comparison to Amorphous RHA Fillers}

The white RHA used in this study is crystalline in nature but by chemical treatment, it may be converted to an amorphous state and undergone some degree of surface modification. Compared to the white RHA, the amorphous RHA (AMRHA) tend to have considerably larger surface area. The BET surface area of the AMRHA was analysed to be 160.5 $\mathrm{m}^{2} \mathrm{~g}^{-1}$ while the surface area of the white RHA is only $1.8 \mathrm{~m}^{2} \mathrm{~g}^{-1}$. Due to the larger surface area, the AMRHA tends to have treater activity than the white RHA. The particle size of the AMRHA $(5.6 \mu \mathrm{m})$ remains quite similar to that of the WRHA $(6.6 \mu \mathrm{m})$. The X-ray diffractogram confirms the amorphous nature of the AMRHA (Figure 2d).

The mechanical properties of the AMRHA composites are shown in Figures $10 \mathrm{a}-\mathrm{c}$. The flexural moduli of the AMRHA composites were marginally better than those of the WRHA but the tensile strength of the former was substantially higher particulary at the maximum $40 \%$ filler loading. This gives a good indication as to the big potential of the material to serve as a filler material though it has less advantage in term of production cost compared to the untreated RHA.

\section{CONCLUSIONS}

It has been shown that even without any coupling agent, the mechanical properties of the RHA composites are not much inferior when compared to the commercial Neuburg silica filler. The RHA fillers have achieved their primary function, i.e., improvement in the modulus of the polypropylene composites without catastrophic effects to the other mechanical properties of interest. Theoretical treatment of the mechanical results has also been successfully applied using the Lewis and Nielsen and Nicolai and Nicodemo models. It is forseen that application of a suitable coupling agent, the interfacial adhesion between the RHA fillers and the polypropylene matrix may be improved leading to superior overall mechanical properties.

\section{REFERENCES}

1) D. V. Rosato, "Rosato's Plastics Encyclopedia and Dictionary," Carl Hanser Verlag, Munich, 1993.

2) M. Y. A. Fuad, J. Mustafah, Z. A. M. Ishak, and A. K. M. Omar, Intern. J. Polym. Mater., 19, 75 (1993).

3) S. S. Chen, SIRIM Technical Report, JKP/33/5-2, 1989.

4) D. O. Hummel and F. Scholl, "Atlas of Polymer and Plastics Analysis," Carl Hanser Verlag, Munich, 1988.

5) E. Guth, J. Appl. Phys., 16, 20 (1945).

6) E. H. Kerner, Proc. Phys. Soc. B., 69, 808 (1956).

7) T. B. Lewis and L. E. Nielsen, J. Appl. Polym. Sci., 14, 1449 (1970).

8) H. A. Schaeffer, "Silica. Encyclopedia of Materials Science and Engineering," M. B. Bever, Ed., Pergamon Press, Oxford, 1986, p 4393.

9) L. Jilken, G. Malhammar, and R. Selden, Polym. Test., 10, 329 (1991).

10) D. M. Bigg, Polym. Composites, 8, 115 (1987).

11) L. E. Nielsen, J. Appl. Polym. Sci., 10, 97 (1966).

12) L. Nicolais and M. Narkis, Polym. Eng. Sci., 11, 194 (1971).

13) L. Nicolais and L. Nicodemo, Polym. Eng. Sci., 13, 469 (1973).

14) J. Leidner and R. T. Woodhams, J. Appl. Polym. Sci., 18, 1639 (1974b).

15) J. A. Manson and L. H. Sperling, "Polymer Blends and Composites," Plenum Press, New York, N.Y., 1976.

16) A. M. Riley, C. D. Paynter, P. M. McGenity, and J. M. Adams, Plast. Rubber Process. Appl., 14, 85 (1990).

17) J. W. Ess and Hornsby, Plast. Rubber Process. Appl., 8, 147 (1987). 